\title{
Heterosis and Combining Ability Studies in Sweet Sorghum [Sorghum bicolor (L.) Moench] for Green Fodder Yield and Its Contributing Traits
}

\author{
T. Soujanya ${ }^{1^{*}}$, T. Shashikala ${ }^{2}$ and A.V. Umakanth ${ }^{3}$ \\ ${ }^{1}$ Department of Genetics and Plant breeding, College of Agriculture, PJTSAU, Telangana, India \\ ${ }^{2}$ AICRP on Forage Crops, ARI, Rajendranagar, Hyderabad, 500030, Telangana, India \\ ${ }^{3}$ ICAR-Indian Institute of Millets Research, Hyderabad 500 030, Telangana, India \\ *Corresponding author
}

\section{A B S T R A C T}

\begin{tabular}{|l|}
\hline K e y w o r d s \\
Standard heterosis, \\
Combining ability, \\
Sweet sorghum, \\
Green fodder yield. \\
\hline Article Info \\
\hline $\begin{array}{l}\text { Accepted: } \\
\text { 28 September } 2017 \\
\text { Available Online: } \\
\text { 10 October } 2017\end{array}$
\end{tabular}

\begin{abstract}
An experiment was conducted to exploit heterosis in $\mathrm{F}_{1}$ hybrids of sweet sorghum (Sorghum bicolor L.) and to identify parents with desirable genetic effects with respect to single-cut green fodder yield and its component traits. $48 \mathrm{~F}_{1}$ hybrids were evaluated to assess general and specific combining ability and gene action governing the green fodder yield and its attributing traits. Analysis of variance revealed the presence of sufficient variation in the experimental material. The SCA variance was greater in magnitude than GCA variance for most of the traits under study indicating predominance of non-additive gene action in the genetic control of those traits. Line 185A and testers RSSV138-1, RSSV466 and RSSV404 emerged as good general combiners for single-cut green fodder yield. Based on sca effects, 185A x RSSV466, 185A x RSSV138-1, PMS71A x RSSV138-1 and PMS71A x RSSV404 were identified as good specific combiners for green fodder yield and its attributing traits.185A x RSSV466 recorded maximum singlecut green fodder yield with 12.53 per cent heterosis over the best check CSV30F.
\end{abstract}

\section{Introduction}

Agricultural crops and livestock play a vital role in the national economy since they fulfill the basic needs of life. Agriculture accounts for $54.6 \%$ of total employment in India and contributes $15.2 \%$ of total GDP. Livestock occupies a crucial position in Indian agriculture and directly contributes $27 \%$ of agricultural GDP. India, with $2.29 \%$ of the world land area, is maintaining about $10.71 \%$ of world's livestock population. The number of milch animals has increased from 62 million in 2000 to 83.15 million in 2012 resulting in $4.04 \%$ year-on-year growth rate of milk (Livestock census, 2012).
Thus, to sustain this growth rate and for further expansion to meet the demands of ever growing human population, livestock needs sustainable supply of feed material.

The area under fodder cultivation is estimated to be about $4 \%$ of the gross cropped area which remained static for the last four decades. The available fodder production is less than the actual requirement. At present, the country faces a net deficit of $61.1 \%$ green fodder, $21.9 \%$ dry crop residues and $64 \%$ concentrate feeds (Dhananjoy, 2013). Moreover, livestock population survives to a 
large extent on crop residues, which are nutritionally poor.

In India, Sorghum (Sorghum bicolor (L.) Moench) is one of the most important forage crops grown widely in north western states and to a limited scale in central and southern states. Sorghum ranks first among the cereal fodder crops because of its growing ability in poor soil, faster growing habit, high yield potential, suitability to cultivate throughout the year, palatability, nutritious fodder quality, higher digestibility and various forms of its utilization. It gives uniform green fodder throughout the year and produces tonnage of dry matter having digestible nutrients $(50 \%)$, crude protein $(8 \%)$, fat $(2.5 \%)$ and nitrogen free extracts (45\%) (Azam et al., 2010). The cultivated area under different forage crops is 4.4 per cent of the total area under cultivation, of which about $2.3 \mathrm{~m}$ ha is under forage sorghum (Anon., 2011).

Sweet sorghum, being a well-known crop can supply food, feed, fodder, fiber and fuel. However, it has not been studied much as a fodder crop. Sweet sorghum has high biomass production, high brix percentage, short duration, low water requirement and wider adaptability (Reddy et al., 2005). Sweet sorghum hybrids have been reported to produce higher sugar yield $(21 \%)$ and higher grain yield $(15 \%)$ than non-sweet sorghum hybrids in the rainy season indicating that there is no trade-off between grain and sugar. The palatability and quality of forage will increase by increasing the sugar content of sorghum stalk. Therefore, the important goals of sweet sorghum forage breeding programs are to increase sweetness, leafiness and juiciness in sorghum (Poehlman, 2006) which can be achieved by developing fodder varieties/hybrids in sweet sorghum with high green fodder yield per unit area and time combined with superior quality. Therefore, the present study was undertaken to assess the general and specific combining ability for fodder yield and quality and to identify parents and crosses with desirable genetic effects and also to assess the possibility of commercial exploitation of heterosis for single-cut green fodder yield and quality through estimating of heterosis over better parent and standard check.

\section{Materials and Methods}

The present investigation involving four lines, namely 185A, ICS38A, 24A, PMS71A twelve testers PMS130, KR135, SSV74, SSVV84, NSSV14, RSSV138-1, RSSV404, RSSV466, IS18542, 6NRL, BNM16, UK81 and forty eight $F_{1}$ hybrids along with the standard check CSV30F were sown in randomized block design with three replications at the research farm of AICRP on Forage Crops, Agricultural Research Institute, Rajendranagar, Hyderabad, Telangana during kharif, 2016. Each entry was raised in two rows of $4 \mathrm{~m}$ length with a spacing of $30 \mathrm{~cm}$ between the rows and $10 \mathrm{~cm}$ between the plants with in the row. All the recommended agronomical practices under AICRP on sorghum were followed and plant protection measures were applied as and when required to ensure good crop. The observations were recorded on five randomly selected plants per each entry in each replication for days to 50 per cent flowering, plant height, number of leaves per plant, leaf length, leaf breadth, number of nodes per plant, internodal length, stem girth, leaf to stem ratio, green fodder yield. Mean of five plants for each entry for each character was calculated and the data was analyzed statistically using the software WINDOSTAT version 8.1.

\section{Results and Discussion}

Analysis of variance for combining ability revealed that parents and crosses differed significantly for all the characters studied (Table 1). The mean squares due to lines and 
testers were significant for most of the characters. Estimates of mean squares for lines and testers revealed the presence of great deal of diversity among the parents with respect to fodder yield and yield contributing traits. The mean squares due to Lines vs Testers were significant for all the characters. This revealed that lines and testers interacted and pronounced different heterotic effects. This could be due to the fact that parents used in this investigation had considerable genetic variability. Analysis of variance for combining ability for yield and yield components indicated that general combining ability (GCA) and specific combining ability (SCA) mean squares were highly significant for all the characters.

The magnitude of sca variance is higher than that of $g c a$ variance for all the traits under study except days to 50 per cent flowering (Mohammed, 2009), (Table 2) and revealed that non additive gene action was predominant in the inheritance of green fodder yield and its component traits. Similar observations were also reported by Bhatt and Bhasketi (2011), Kamdi et al., (2011), Akbari et al., (2013) and Tariq et al., (2014).

The range of standard heterosis of different characters along with number of significant crosses are presented in the Table 3 . In the present study, magnitude of heterosis varied from cross to cross for all the characters studied. Standard heterosis is the most effective parameter amongst the three parameters of heterosis. In the present investigation, well known variety CSV30F is used as standard check to get the information on superiority of the crosses. Considerable amount of heterosis was observed for green fodder yield and its component traits. The results revealed presence of heterosis for majority of the traits under study. The hybrid, 185A x RSSV466 exhibited high estimates of standard heterosis for green fodder yield and also showed heterosis in desirable direction for characters like for days to 50 per cent flowering and leaf breadth. Hybrid 27A x RSSV138-1 showed heterosis in desirable direction for characters like, plant height, leaf breadth, internodal length and stem girth. 185A x RSSV138-1 showed heterosis in desirable direction for number of leaves per plant and leaf breadth, 27A x RSSV466 showed heterosis in desirable direction for days to 50 per cent flowering, number of leaves per plant and leaf breadth. ICS38A x IS18542 showed heterosis in desirable direction for leaf length, leaf breadth and internodal length.

In the present study, hybrid 185A x RSSV466 exhibited highest significant positive heterosis of 12.53 per cent over the best check with highest green fodder yield of $62.47 \mathrm{t} \mathrm{ha}^{-1}$. In addition, the hybrid also exhibited significant heterosis in desirable direction for days to 50 per cent flowering and leaf breadth.

The general combining ability effects of lines and testers are presented in the Table 4. Among the parents, 185A showed significant gca effects in desirable direction for days to 50 per cent flowering, green fodder yield leaf breadth and stem girth. RSSV138-1 was found good general combiner for green fodder yield, plant height, number of leaves per plant, leaf length, leaf breadth and number of nodes per plant. RSSV466 showed high significant desirable $\mathrm{gca}$ effects for days to 50 per cent flowering, green fodder yield, plant height, leaf breadth and number of nodes per plant. RSSV404 showed high significant desirable gca effects for green fodder yield, plant height, number of leaves per plant, number of nodes per plant and leaf to stem ratio. High GCA values of parents RSSV1381, RSSV466, RSSV404 and 185A for green fodder yield and its contributing traits indicated that these parents had favourable genes, therefore could be better choices for improvement of forage yield and component traits through hybridization. 
Table.1 Analysis of variance for green fodder yield and yield components in sweet sorghum

\begin{tabular}{|c|c|c|c|c|c|c|c|c|c|c|c|}
\hline $\begin{array}{l}\text { Source of } \\
\text { variation }\end{array}$ & d.f. & $\begin{array}{c}\text { Days to } \\
50 \% \\
\text { flowering }\end{array}$ & Plant height & $\begin{array}{l}\text { No of } \\
\text { leaves } \\
\text { /plant }\end{array}$ & $\begin{array}{c}\text { Leaf } \\
\text { length }\end{array}$ & $\begin{array}{c}\text { Leaf } \\
\text { breadth }\end{array}$ & $\begin{array}{c}\text { No of } \\
\text { nodes / } \\
\text { plant }\end{array}$ & $\begin{array}{c}\text { Internodal } \\
\text { length }\end{array}$ & $\begin{array}{l}\text { Stem } \\
\text { girth }\end{array}$ & $\begin{array}{c}\text { Leaf to } \\
\text { stem } \\
\text { ratio }\end{array}$ & $\begin{array}{c}\text { Green } \\
\text { fodder } \\
\text { yield }\end{array}$ \\
\hline Replications & 2 & 1.764 & 1.515 & 0.226 & 47.738 & 0.016 & 0.004 & 0.231 & 0.003 & 0.094 & 4.876 \\
\hline Crosses & 47 & $186.541 * *$ & $2410.438 * *$ & $5.038 * *$ & $172.985 * *$ & $1.022 * *$ & $2.529 * *$ & $18.689 * *$ & $0.692 * *$ & $15.002 * *$ & $262.145 * *$ \\
\hline Lines & 3 & 1316.206 & $11776.320 * *$ & 4.326 & $796.594 * *$ & $3.998 * *$ & $7.325 * *$ & $40.188 * *$ & $3.275 * *$ & $80.357 * *$ & $733.290 * *$ \\
\hline Testers & 11 & 216.647 & $3870.859 * *$ & $14.313 * *$ & 143.020 & 0.654 & $4.742 * *$ & $43.525 * *$ & $1.067 * *$ & 12.968 & $456.227 * *$ \\
\hline Line*Tester & 33 & $73.809 * *$ & $1072.188 * *$ & $2.011 * *$ & $126.281 * *$ & $0.889 * *$ & $1.356 * *$ & $8.456 * *$ & $0.333 * *$ & $9.739 * *$ & $154.619 * *$ \\
\hline Error & 94 & 16.255 & 1.218 & 0.135 & 46.732 & 0.010 & 0.015 & 0.129 & 0.011 & 0.274 & 7.155 \\
\hline Total & 143 & 51.911 & 793.064 & 1.748 & 88.242 & 0.343 & 0.841 & 6.230 & 0.235 & 5.112 & 90.931 \\
\hline
\end{tabular}

*significant at $5 \%$ level, **significant at $1 \%$ level

Table.2 Estimation of general and specific combining ability variances for green fodder yield and its components in sweet sorghum

\begin{tabular}{|c|c|c|c|c|c|c|c|c|c|c|}
\hline $\begin{array}{l}\text { Source of } \\
\text { variation }\end{array}$ & $\begin{array}{c}\text { Days to } \\
50 \% \\
\text { flowering }\end{array}$ & $\begin{array}{c}\text { Plant } \\
\text { height }\end{array}$ & $\begin{array}{l}\text { No of } \\
\text { leaves } \\
\text { /plant }\end{array}$ & $\begin{array}{c}\text { Leaf } \\
\text { length }\end{array}$ & $\begin{array}{c}\text { Leaf } \\
\text { breadth }\end{array}$ & $\begin{array}{l}\text { No of } \\
\text { nodes } \\
\text { /plant }\end{array}$ & $\begin{array}{c}\text { Internodal } \\
\text { length }\end{array}$ & $\begin{array}{l}\text { Stem } \\
\text { girth }\end{array}$ & $\begin{array}{c}\text { Leaf to } \\
\text { stem ratio }\end{array}$ & $\begin{array}{c}\text { Green } \\
\text { fodder } \\
\text { yield }\end{array}$ \\
\hline$\sigma^{2}$ gca & 31.302 & 325.591 & 0.383 & 18.115 & 0.0961 & 0.251 & 1.737 & 0.090 & 1.931 & 24.478 \\
\hline$\sigma^{2}$ sca & 19.544 & 354.258 & 0.625 & 30.408 & 0.289 & 0.447 & 2.765 & 0.105 & 3.141 & 49.114 \\
\hline $\begin{array}{c}\sigma^{2} \text { gca / } \\
\sigma^{2} \text { sca }\end{array}$ & 1.60 & 0.919 & 0.612 & 0.602 & 0.331 & 0.561 & 0.628 & 0.857 & 0.614 & 0.498 \\
\hline
\end{tabular}


Table.3 The range of standard heterosis and number of crosses showing significant heterosis for various traits in sweet sorghum

\begin{tabular}{|c|c|c|c|}
\hline \multirow[t]{2}{*}{ Characters } & \multirow[t]{2}{*}{ Range of heterosis } & \multicolumn{2}{|c|}{$\begin{array}{c}\text { Noumber of hybrids having significan } \\
\text { heterotic effect }\end{array}$} \\
\hline & & + ve & -ve \\
\hline Days to $50 \%$ flowering & $\begin{array}{c}-14.23 \text { to } 15.81 \\
(185 \mathrm{~A} \text { x RSSV466) }\end{array}$ & 16 & 14 \\
\hline Plant height $(\mathrm{cm})$ & $\begin{array}{c}-38.41 \text { to } 6.22 \\
(27 \mathrm{~A} \times \mathrm{RSSV} 138-1)\end{array}$ & 2 & 44 \\
\hline No of leaves /plant & $\begin{array}{c}-35.83 \text { to } 11.53 \\
(185 \mathrm{~A} \text { x RSSV138-1) } \\
\end{array}$ & 3 & 43 \\
\hline Leaf length $(\mathrm{cm})$ & $\begin{array}{c}-45.80 \text { to } 11.15 \\
\text { (ICS38A x IS18542) }\end{array}$ & 8 & 16 \\
\hline Leaf breadth $(\mathrm{cm})$ & $\begin{array}{c}-12.18 \text { to } 23.69 \\
(185 \mathrm{~A} x \mathrm{RSSV} 466)\end{array}$ & 31 & 2 \\
\hline No of nodes /plant & $\begin{array}{c}-34.33 \text { to } 0.89 \\
\text { (PMS71A x RSSV404) }\end{array}$ & 1 & 47 \\
\hline Internodal length & $\begin{array}{c}-29.84 \text { to } 11.55 \\
\text { (PMS71A XPMS130) }\end{array}$ & 2 & 41 \\
\hline Stem girth & $\begin{array}{c}-26.54 \text { to } 7.02 \\
(27 \mathrm{~A} \times \mathrm{BNM} 16)\end{array}$ & 3 & 31 \\
\hline Leaf to stem ratio & $\begin{array}{c}-36.32 \text { to } 0.82 \\
\text { (ICS38A } x \text { KR135) }\end{array}$ & 1 & 45 \\
\hline Green fodder yield (t/ha) & $\begin{array}{c}-67.53 \text { to } 12.53 \\
(185 \mathrm{~A} \times \mathrm{RSSV} 466\end{array}$ & 1 & 47 \\
\hline
\end{tabular}

Table.4 General combining ability effects of parents for green fodder yield and yield components in sweet sorghum

\begin{tabular}{|c|c|c|c|c|c|c|c|c|c|c|}
\hline Genotype & $\begin{array}{c}\text { Days to } \\
\mathbf{5 0 \%} \\
\text { flowering }\end{array}$ & $\begin{array}{l}\text { Plant } \\
\text { height } \\
(\mathbf{c m})\end{array}$ & $\begin{array}{l}\text { No. of } \\
\text { leaves } \\
\text { /plant }\end{array}$ & $\begin{array}{c}\text { Leaf } \\
\text { length } \\
(\mathrm{cm})\end{array}$ & $\begin{array}{c}\text { Leaf } \\
\text { breadth } \\
(\mathrm{cm})\end{array}$ & $\begin{array}{l}\text { No. of } \\
\text { nodes } \\
\text { /plant }\end{array}$ & $\begin{array}{l}\text { Internodal } \\
\text { length }(\mathrm{cm})\end{array}$ & $\begin{array}{l}\text { Stem } \\
\text { girth } \\
(\mathrm{cm})\end{array}$ & $\begin{array}{c}\text { Leaf to } \\
\text { stem ratio }\end{array}$ & $\begin{array}{c}\text { Green fodder } \\
\text { yield (t/ha) }\end{array}$ \\
\hline $185 \mathrm{~A}$ & $-8.425^{* *}$ & $-22.586^{* *}$ & $-0.182 * *$ & $-6.944 * *$ & $0.440^{* *}$ & $-0.605^{* *}$ & $-0.570 * *$ & $0.311^{* *}$ & -0.071 & $6.120 * *$ \\
\hline ICS38A & 3.025 & $-1.753^{* *}$ & $0.123^{*}$ & $3.361 * *$ & -0.030 & $0.151 * *$ & $-0.711^{* *}$ & -0.023 & $2.133^{* *}$ & $-4.604 * *$ \\
\hline $27 \mathrm{~A}$ & -0.036 & $21.443 * *$ & $0.423 * *$ & $2.270^{*}$ & $-0.049 *$ & $0.470 * *$ & $1.562^{* * *}$ & $0.114 * *$ & $-0.939 * *$ & 0.052 \\
\hline PMS71A & $5.436^{* *}$ & $2.896^{* * *}$ & $-0.365^{* *}$ & 1.313 & $-0.362 * *$ & -0.016 & $-0.282 * *$ & $-0.403 * *$ & $-1.123^{* *}$ & $-1.568 * *$ \\
\hline SE (lines) & 0.9182 & 0.7232 & 0.0873 & 1.3956 & 0.0328 & 0.0960 & 0.0942 & 0.0328 & 0.1325 & 0.6358 \\
\hline PMS130 & -4.525 & $-31.218^{* *}$ & $-1.265^{* *}$ & -2.940 & -0.034 & $-1.170 * *$ & $-1.983^{* *}$ & $0.116^{* *}$ & $1.260 * *$ & -1.313 \\
\hline KR135 & -2.025 & $-25.385^{* *}$ & $-0.487 * *$ & -0.374 & -0.044 & $-0.672 * *$ & $-1.854 * *$ & $0.326 * *$ & $1.312 * *$ & $-3.921 * *$ \\
\hline SSV74 & 1.933 & $2.345^{* *}$ & -0.078 & 0.598 & $-0.118^{* *}$ & 0.066 & $-1.518^{* *}$ & $-0.242 * *$ & $-1.005^{* *}$ & $-5.134 * *$ \\
\hline SSV84 & $4.158^{* *}$ & $-20.195^{* *}$ & $-0.556^{* *}$ & -2.549 & $-0.224 * *$ & $0.068^{*}$ & $-1.498 * *$ & $-0.655^{* *}$ & -0.273 & $-7.210 * *$ \\
\hline NSSV14 & 1.775 & $5.460 * *$ & $-0.739 * *$ & $-4.365^{*}$ & $-.284 * *$ & 0.053 & $-1.333 * *$ & $-0.174 * *$ & $-1.238 * *$ & $-2.694 *$ \\
\hline RSSV138-1 & $7.317 * *$ & $29.555^{* *}$ & $2.550 * *$ & $8.048 * *$ & $0.147 * *$ & $1.146^{* *}$ & $0.936^{* * *}$ & -0.074 & $-1.441 * *$ & $4.586^{* *}$ \\
\hline RSSV404 & 0.075 & $17.375^{* *}$ & $0.736^{* *}$ & 1.516 & 0.047 & $0.793 * *$ & $2.045^{* *}$ & 0.005 & $0.562 * *$ & $8.023 * *$ \\
\hline RSSV466 & $-6.425 * *$ & $6.689 * *$ & -0.002 & $-3.959^{*}$ & $0.474 * *$ & $0.121 * *$ & $3.350^{* * *}$ & 0.076 & -0.039 & $11.874 * *$ \\
\hline IS18542 & $4.658^{* * *}$ & $5.872 * *$ & $1.545^{* *}$ & 2.771 & $0.233^{* *}$ & $0.507 * *$ & $-0.374 * *$ & $0.377 * *$ & $0.690 * *$ & $-5.053 * *$ \\
\hline $6 \mathrm{NRL}$ & $-4.842 * *$ & $14.251 * *$ & $-0.557 * *$ & 1.706 & $0.171^{* *}$ & $-0.161 * *$ & $2.826^{* * *}$ & $0.336^{* *}$ & $-0.458 * *$ & $-4.614 * *$ \\
\hline BNM16 & -3.008 & $-5.307 * *$ & $-0.470 * *$ & 0.991 & -0.049 & $-0.518 * *$ & $0.602 * *$ & $0.142 * *$ & $1.473 * *$ & $6.430 * *$ \\
\hline UK81 & 0.908 & 0.560 & $-0.677 * *$ & -1.444 & $0.316^{* *}$ & $-0.097 * *$ & $-1.197 * *$ & $-0.231 * *$ & $-0.844 * *$ & -0.973 \\
\hline SE (testers) & 1.5903 & 1.2526 & 0.1512 & 2.4172 & 0.0568 & 0.0480 & 0.1632 & 0.0569 & 0.2296 & 1.1012 \\
\hline
\end{tabular}

*significant at $5 \%$ level, **significant at $1 \%$ level 
Table.5 Specific combining ability effects for for green fodder yield and yield components in sweet sorghum

\begin{tabular}{|c|c|c|c|c|c|c|c|c|c|c|}
\hline Genotype & $\begin{array}{c}\text { Days to } \\
50 \% \\
\text { flowering }\end{array}$ & $\begin{array}{l}\text { Plant } \\
\text { height } \\
(\mathrm{cm})\end{array}$ & $\begin{array}{l}\text { No of } \\
\text { leaves } \\
\text { /plant }\end{array}$ & $\begin{array}{c}\text { Leaf } \\
\text { length } \\
(\mathrm{cm})\end{array}$ & $\begin{array}{c}\text { Leaf } \\
\text { breadth } \\
(\mathrm{cm})\end{array}$ & $\begin{array}{c}\text { No of } \\
\text { nodes/pla } \\
\text { nt }\end{array}$ & $\begin{array}{l}\text { Internodal } \\
\text { length }(\mathrm{cm})\end{array}$ & $\begin{array}{l}\text { Stem } \\
\text { girth } \\
(\mathrm{cm})\end{array}$ & $\begin{array}{c}\text { Leaf to } \\
\text { stem ratio }\end{array}$ & $\begin{array}{c}\text { Green } \\
\text { fodder } \\
\text { yield } \\
\text { (t/ha) }\end{array}$ \\
\hline $185 \mathrm{~A} \times \mathrm{PMS} 130$ & 1.125 & $16.305^{* *}$ & $0.767 * *$ & 1.283 & 0.061 & $0.681 * *$ & $0.678 * *$ & -0.107 & $-3.349 * *$ & $-9.080 * *$ \\
\hline 185A x KR135 & 3.292 & $-9.954 * *$ & $-1.007 * *$ & -2.374 & $-0.982 * *$ & $-0.327 * *$ & $-1.038 * *$ & $-0.371 * *$ & $-1.494 * *$ & -2.915 \\
\hline 185A x SSV74 & 0.067 & $-42.158 * *$ & $-1.479 * *$ & 1.561 & $-0.438 * *$ & $-1.031 * *$ & $-1.290 * *$ & $-0.200^{*}$ & -0.177 & 0.645 \\
\hline 185A x SSV84 & -2.158 & $-20.241 * *$ & $-0.968 * *$ & -3.205 & $-1.262 * *$ & $-1.201 * *$ & $-1.555 * *$ & $-0.170^{*}$ & -0.566 & 0.987 \\
\hline $185 \mathrm{~A} \times \mathrm{NSSV} 14$ & -3.308 & $-18.806 * *$ & 0.182 & $-20.292 * *$ & $0.848 * *$ & $0.435^{* *}$ & $-1.259 * *$ & $0.616^{* *}$ & $1.989 * *$ & $6.198 * *$ \\
\hline 185A x RSSV138-1 & $12.017 * *$ & $22.756 * *$ & $1.576^{* *}$ & $10.698 * *$ & $0.314 * *$ & $0.379 * *$ & $3.759 * *$ & $0.349 * *$ & -0.181 & $-10.809 * *$ \\
\hline 185A x RSSV404 & -3.742 & $-10.888 * *$ & 0.140 & 2.926 & $0.300 * *$ & -0.011 & $-1.404 * *$ & 0.070 & $1.530 * *$ & -0.480 \\
\hline $185 \mathrm{~A}$ x RSSV466 & 0.758 & $31.882 * *$ & $1.078 * *$ & $7.315^{*}$ & $0.170 *$ & $0.730 * *$ & $1.925 * *$ & $-0.211 *$ & 0.231 & $12.473 * *$ \\
\hline 185A x IS18542 & $-7.992 * *$ & -2.752 & $-1.303 * *$ & -4.089 & -0.086 & $-0.175^{*}$ & $0.692 * *$ & -0.112 & $1.235 * *$ & $-3.640^{*}$ \\
\hline 185A x6NRL & 2.842 & $27.236^{* *}$ & 0.367 & 6.774 & $0.470 * *$ & $1.392 * *$ & 0.322 & -0.061 & $1.186^{* *}$ & $3.958 *$ \\
\hline $185 \mathrm{~A} \times \mathrm{BNM} 16$ & 0.008 & $7.894 * *$ & 0.296 & -2.849 & $0.266^{* *}$ & -0.131 & $-1.488 * *$ & -0.121 & $-1.105 * *$ & $-5.986 * *$ \\
\hline 185A x UK81 & -2.908 & -1.273 & 0.352 & 2.253 & $0.340 * *$ & 0.131 & $0.658 * *$ & $0.316^{* *}$ & $0.702 *$ & $8.650 * *$ \\
\hline ICS38A x PMS130 & -1.258 & $16.672 * *$ & -0.080 & -2.121 & $-0.262 * *$ & $-0.372 * *$ & $1.029 * *$ & $0.203^{*}$ & 0.328 & $15.547 * *$ \\
\hline ICS38A x KR135 & -2.425 & $-7.360 * *$ & -0.275 & -2.288 & -0.049 & $-0.427 * *$ & -0.067 & 0.100 & 0.622 & $-4.605 * *$ \\
\hline ICS38A x SSV74 & $-5.617 *$ & $17.875 * *$ & $0.963 * *$ & $-8.110 *$ & $0.212 * *$ & $0.505^{* *}$ & $-0.666 * *$ & $0.308 * *$ & 0.180 & $3.308 *$ \\
\hline ICS38A x SSV84 & 0.725 & $-5.950 * *$ & $0.960 * *$ & 4.571 & $0.531 * *$ & $1.409 * *$ & $-1.787 * *$ & 0.067 & -0.483 & -2.163 \\
\hline ICS38A x NSSV14 & 4.108 & $4.761 * *$ & -0.066 & $9.537 * *$ & -0.149 & $-0.592 * *$ & $2.476^{* *}$ & $0.190 *$ & $-1.881 * *$ & -2.505 \\
\hline ICS38A x RSSV138-1 & $-6.133 * *$ & $-32.944 * *$ & $-1.005 * *$ & -6.056 & 0.107 & 0.025 & $-1.620 * *$ & -0.117 & $0.909 * *$ & -2.855 \\
\hline ICS38A x RSSV404 & -2.192 & $-6.527 * *$ & $-1.221 * *$ & -4.178 & -0.030 & $-0.428 * *$ & $-0.529 *$ & $-0.323 * *$ & -0.247 & 0.651 \\
\hline ICS38A x RSSV466 & 3.975 & $-15.068 * *$ & -0.333 & -3.269 & $-0.450 * *$ & $-0.496 * *$ & $-2.994 * *$ & 0.089 & -0.263 & $-7.387 * *$ \\
\hline ICS38A x IS18542 & 3.558 & -1.518 & $1.490 * *$ & $9.964 * *$ & $0.560 * *$ & 0.115 & $0.850 * *$ & 0.148 & $-1.282 * *$ & 2.623 \\
\hline ICS38A x x6NRL & 4.058 & $14.103 * *$ & 0.015 & 2.999 & -0.107 & $-0.178 *$ & $1.890^{*}$ & -0.133 & $-1.394 * *$ & 0.102 \\
\hline ICS38A x BNM16 & 2.558 & 1.428 & -0.092 & -1.893 & $-0.314 * *$ & -0.125 & $0.613 * *$ & $-0.180^{*}$ & $3.308 * *$ & 0.837 \\
\hline ICS38A x UK81 & -1.358 & $14.528 * *$ & -0.355 & 0.845 & -0.050 & $0.565 * *$ & $0.806 * *$ & $-0.353 * *$ & 0.202 & $-3.553 *$ \\
\hline 27A x PMS130 & $-4.864 *$ & $-7.877 * *$ & $-0.598 * *$ & 4.213 & $1.058 * *$ & 0.093 & 0.093 & $0.187^{*}$ & $0.670^{*}$ & 1.157 \\
\hline 27A x KR135 & -4.031 & $12.651 * *$ & $0.625 * *$ & 0.903 & $0.65 * *$ & $0.481 * *$ & 0.430 & $0.387 * *$ & 0.598 & $11.432 * *$ \\
\hline 27A x SSV74 & 3.344 & $10.990 * *$ & 0.139 & 5.868 & -0.155 & $0.733 * *$ & $0.785 * *$ & $-0.402 * *$ & $1.192 * *$ & -2.222 \\
\hline $27 \mathrm{~A} \times \mathrm{SSV} 84$ & 1.119 & $27.254 * *$ & $0.527 *$ & 1.879 & $0.478 * *$ & $0.323 * *$ & $1.114 * *$ & 0.094 & -0.517 & -1.972 \\
\hline $27 \mathrm{~A} \times \mathrm{NSSV} 14$ & 2.169 & 0.066 & 0.020 & 6.621 & $0.892 * *$ & $0.683 * *$ & $-1.804 * *$ & $-0.687 * *$ & 0.138 & -1.618 \\
\hline 27A x RSSV138-1 & 1.294 & $7.537 * *$ & 0.187 & -3.879 & -0.130 & $-0.557 * *$ & $-1.760 * *$ & -0.037 & -0.272 & -1.942 \\
\hline $27 \mathrm{~A} \times \mathrm{RSSV} 404$ & $8.869 * *$ & $11.751 * *$ & $1.002 * *$ & -3.221 & -0.120 & $-0.550 * *$ & $0.491 *$ & $-0.239 * *$ & $-1.942 * *$ & $-8.879 * *$ \\
\hline 27A x RSSV466 & -2.297 & $-6.763 * *$ & -0.237 & -3.758 & 0.139 & 0.062 & $1.893 * *$ & $0.303 * *$ & $2.216^{* *}$ & $-3.703^{*}$ \\
\hline 27A x IS18542 & $5.953 * *$ & -0.247 & $-0.474 *$ & -2.124 & $-0.610 * *$ & 0.073 & $-0.650 * *$ & $-0.235 * *$ & $-3.537 * *$ & 1.427 \\
\hline $27 \mathrm{~A} \times 6 \mathrm{NRL}$ & $-6.881 * *$ & $-31.693 * *$ & $-0.472 *$ & $-9.253 * *$ & -0.044 & $-0.703 * *$ & $-1.640 * *$ & $0.173 *$ & $1.961 * *$ & 3.058 \\
\hline 27A x BNM16 & $-5.381 *$ & $-7.334 * *$ & $-0.526^{*}$ & -4.081 & 0.049 & $0.193^{* *}$ & $1.774 * *$ & $0.653 * *$ & -0.453 & $7.318^{* *}$ \\
\hline 27A x UK81 & 0.703 & $-16.334 * *$ & -0.103 & -1.041 & $-0.424 * *$ & $-0.831 * *$ & $-0.727 * *$ & $-0.197 *$ & -0.052 & $-4.056^{*}$ \\
\hline PMS71A x PMS130 & $4.997 *$ & $-25.100 * *$ & -0.089 & -3.374 & $-0.857 * *$ & $-0.402 * *$ & $-1.800 * *$ & $-0.283 * *$ & $2.350 * *$ & $-7.623 * *$ \\
\hline PMS71A x KR135 & 3.164 & $4.664 * *$ & 0.657 ** & 3.759 & $0.380 * *$ & $0.273 * *$ & $0.674 * *$ & -0.116 & 0.275 & $-3.911 *$ \\
\hline PMS71A x SSV74 & 2.206 & $13.293 * *$ & 0.377 & 0.681 & $0.381 * *$ & $-0.207 * *$ & $1.172 * *$ & $0.295 * *$ & $-1.195 * *$ & -1.731 \\
\hline PMS71A x SSV84 & 0.314 & -1.063 & $-0.518^{*}$ & -3.245 & $0.253 * *$ & $-0.531 * *$ & $2.228 * *$ & 0.008 & $1.566 * *$ & $3.148^{*}$ \\
\hline PMS71A x NSSV14 & -2.969 & $13.979 * *$ & -0.135 & 4.134 & $0.193 *$ & $0.345 * *$ & $0.587 *$ & -0.119 & -0.245 & -2.075 \\
\hline PMS71A x RSSV138-1 & $-7.178 * *$ & 2.651 & $-0.758 * *$ & -0.762 & $-0.291 * *$ & $0.153^{*}$ & -0.379 & $-0.196^{*}$ & -0.455 & $15.605^{* *}$ \\
\hline PMS71A x RSSV404 & -2.936 & $5.664 * *$ & 0.080 & 4.473 & -0.151 & $0.989 * *$ & $1.442 * *$ & $0.491 * *$ & $0.659 *$ & $8.708 * *$ \\
\hline PMS71A x RSSV466 & -2.436 & $-10.050 * *$ & -0.418 & -0.288 & 0.142 & $-0.296 * *$ & $-0.823 * *$ & $-0.180^{*}$ & $-2.184 * *$ & -1.383 \\
\hline PMS71A x IS18542 & -1.519 & $4.517 *$ & 0.287 & -3.462 & 0.136 & -0.012 & $-0.892 * *$ & $0.199 *$ & $3.584 * *$ & -0.410 \\
\hline PMS71A x x6NRL & -0.019 & $-9.646 * *$ & 0.090 & -0.520 & $-0.318 * *$ & $-0.511 * *$ & $-0.572 *$ & 0.021 & $-1.752 * *$ & $-7.118 * *$ \\
\hline PMS71A x BNM16 & 2.814 & -1.988 & 0.322 & 0.661 & -0.002 & 0.063 & $-0.899 * *$ & $-0.353 * *$ & $-1.750 * *$ & -2.169 \\
\hline PMS71A x UK81 & 3.564 & 3.079 & 0.106 & -2.057 & 0.135 & $0.135^{*}$ & $-0.737 * *$ & $0.234 * *$ & $-0.852 *$ & -1.042 \\
\hline SE (crosses) & 3.1806 & 2.5051 & 0.3024 & 4.8344 & 0.1137 & 0.0960 & 0.3264 & 0.1137 & 0.4591 & 2.2024 \\
\hline
\end{tabular}


Table.6 Top ranking desirable crosses for sca effects with their per se performance

\begin{tabular}{|c|c|c|c|c|c|c|}
\hline \multirow[t]{2}{*}{ Character and Cross } & \multirow{2}{*}{$\begin{array}{l}\text { Predominant } \\
\text { gene action }\end{array}$} & \multirow{2}{*}{$\begin{array}{l}\text { Values of } \\
\text { sca effects }\end{array}$} & \multirow[t]{2}{*}{$\begin{array}{l}\text { gca status of } \\
\text { parents }\end{array}$} & \multicolumn{2}{|c|}{$\begin{array}{c}\text { Values of } g c a \text { effects } \\
\text { of parents }\end{array}$} & \multirow{2}{*}{$\begin{array}{c}\text { Per se } \\
\text { performance }\end{array}$} \\
\hline & & & & $\mathrm{P}-1$ & $P-2$ & \\
\hline Days to $50 \%$ flowering & Additive & & & & & \\
\hline $185 \mathrm{~A}$ x IS18542 & & -7.992 & $\mathrm{H} \times \mathrm{L}$ & -8.425 & 4.658 & 74.67 \\
\hline PMS71A x RSSV138-1 & & -7.178 & $\mathrm{~L} \times \mathrm{L}$ & 5.436 & 7.317 & 92 \\
\hline $27 \mathrm{~A} \times 6 \mathrm{NRL}$ & & -6.881 & $\mathrm{~L} \times \mathrm{H}$ & -0.036 & -4.84 & 74.67 \\
\hline ICS38A x RSSV138-1 & & -6.133 & $\mathrm{~L} \times \mathrm{L}$ & 3.025 & 7.317 & 90.67 \\
\hline Plant height $(\mathrm{cm})$ & Non additive & & & & & \\
\hline $185 \mathrm{~A} \times \mathrm{RSSV} 466$ & & 31.882 & $\mathrm{~L} \times \mathrm{H}$ & -22.586 & 6.689 & 246.78 \\
\hline $27 \mathrm{~A} \times \mathrm{SSV} 84$ & & 27.254 & $\mathrm{H} \times \mathrm{L}$ & 21.443 & -20.195 & 259.30 \\
\hline $185 \mathrm{~A} \times 6 \mathrm{NRL}$ & & 27.236 & $\mathrm{~L} \times \mathrm{H}$ & -22.586 & 14.251 & 249.70 \\
\hline $185 \mathrm{~A} \times \mathrm{RSSV} 138-1$ & & 22.756 & $\mathrm{~L} \times \mathrm{H}$ & -22.586 & 29.55 & 260.52 \\
\hline Number of leaves /plant & Non additive & & & & & \\
\hline $185 \mathrm{~A} \times \mathrm{RSSV} 138-1$ & & 1.576 & $\mathrm{~L} \times \mathrm{H}$ & -0.182 & 2.550 & 13.38 \\
\hline ICS38A x IS18542 & & 1.490 & $\mathrm{H} \times \mathrm{H}$ & 0.123 & 1.545 & 12.60 \\
\hline $185 \mathrm{~A} \times \mathrm{RSSV} 466$ & & 1.078 & $\mathrm{~L} \times \mathrm{L}$ & -0.182 & -0.002 & 10.33 \\
\hline Leaf length $(\mathrm{cm})$ & Non additive & & & & & \\
\hline $185 \mathrm{~A} \times \mathrm{RSSV} 138-1$ & & 10.698 & $\mathrm{~L} \times \mathrm{H}$ & -6.994 & 8.048 & 88.80 \\
\hline ICS38A x IS18542 & & 9.964 & $\mathrm{H} \times \mathrm{L}$ & 3.361 & 2.771 & 93.10 \\
\hline ICS38A x NSSV14 & & 9.537 & $\mathrm{H} \times \mathrm{L}$ & 3.361 & -4.365 & 85.53 \\
\hline $185 \mathrm{~A}$ x RSSV466 & & 7.315 & $\mathrm{~L} \times \mathrm{L}$ & -6.994 & -3.959 & 73.41 \\
\hline Leaf breadth (cm) & Non additive & & & & & \\
\hline 27A x PMS130 & & 1.058 & $\mathrm{~L} \times \mathrm{L}$ & -0.049 & -0.034 & 7.95 \\
\hline 27A x NSSV14 & & 0.892 & $\mathrm{~L} \times \mathrm{L}$ & -0.049 & -0.284 & 5.75 \\
\hline $185 \mathrm{~A} \times \mathrm{NSSV} 14$ & & 0.848 & $\mathrm{H} \times \mathrm{L}$ & 0.440 & -0.284 & 7.98 \\
\hline Number of nodes/plant & Non additive & & & & & \\
\hline ICS38A x SSV84 & & 1.409 & $\mathrm{H} \times \mathrm{H}$ & 0.151 & 0.068 & 10.51 \\
\hline $185 \mathrm{~A} \times 6 \mathrm{NRL}$ & & 1.392 & $\mathrm{~L} \times \mathrm{L}$ & -0.605 & -0.161 & 9.65 \\
\hline PMS71A x RSSV404 & & 0.989 & $\mathrm{~L} \times \mathrm{H}$ & -0.016 & 0.793 & 10.79 \\
\hline Internodal length (cm) & Non additive & & & & & \\
\hline ICS38A x RSSV466 & & -2.994 & $\mathrm{H} \times \mathrm{L}$ & -0.711 & 3.350 & 22.14 \\
\hline 27A x NSSV14 & & -1.804 & $\mathrm{~L} \times \mathrm{H}$ & 1.562 & -1.333 & 20.92 \\
\hline PMS71A x PMS130 & & -1.800 & $\mathrm{H} \times \mathrm{H}$ & -0.282 & -1.983 & 18.43 \\
\hline 27A x RSSV138-1 & & -1.760 & $\mathrm{~L} \times \mathrm{L}$ & 1.562 & 0.936 & 23.23 \\
\hline Leaf to stem ratio & Non additive & & & & & \\
\hline PMS71A x IS18542 & & 3.584 & $\mathrm{~L} \times \mathrm{H}$ & -1.123 & 0.690 & 0.204 \\
\hline ICS38A x BNM16 & & 0.653 & $\mathrm{H} \times \mathrm{H}$ & 2.133 & 1.473 & 0.241 \\
\hline PMS71A x PMS130 & & 2.350 & $\mathrm{~L} \times \mathrm{H}$ & -1.123 & 1.260 & 0.197 \\
\hline $27 \mathrm{~A} \times \mathrm{RSSV} 466$ & & 2.216 & $\mathrm{~L} \times \mathrm{L}$ & -0.939 & -0.039 & 0.184 \\
\hline Stem girth $(\mathrm{cm})$ & Non additive & & & & & \\
\hline $27 \mathrm{~A} \times \mathrm{BNM} 16$ & & 0.653 & $\mathrm{H} \times \mathrm{H}$ & 0.114 & 0.142 & 2.50 \\
\hline $185 \mathrm{~A} \times \mathrm{NSSV} 14$ & & 0.616 & $\mathrm{H} \times \mathrm{L}$ & 0.311 & -0.174 & 2.44 \\
\hline Green fodder yield & Non additive & & & & & \\
\hline PMS71A x RSSV138-1 & & 15.605 & $\mathrm{~L} \times \mathrm{H}$ & -1.568 & 4.586 & 50.63 \\
\hline ICS38A x PMS130 & & 15.547 & $\mathrm{~L} \times \mathrm{L}$ & -4.604 & -1.313 & 41.63 \\
\hline 185A x RSSV466 & & 12.473 & $\mathrm{H} \times \mathrm{H}$ & 6.120 & 11.874 & 62.47 \\
\hline
\end{tabular}

P-1: Parent-1; P-2: Parent-2

Based on the sca effects (Table 5), the hybrid 185A x RSSV466 exhibited significant SCA effects in desirable direction for green fodder yield, plant height, number of leaves per plant, leaf length, leaf breadth and number of nodes per plant. 185A x RSSV138-1 exhibited significant SCA effects in desirable direction for plant height, number of leaves per plant, leaf length, leaf breadth, number of nodes per plant and stem girth. 185A x RSSV404 exhibited significant SCA effects in desirable direction for leaf breadth, 
intermodal length and leaf to stem ratio. PMS71A x RSSV138-1 exhibited significant SCA effects in desirable direction for green fodder yield, days to 50 per cent flowering, number of nodes per plant. PMS71A $x$ RSSV404 showed significant SCA effects in desirable direction for green fodder yield, plant height, number of nodes per plant, stem girth and leaf to stem ratio. 27A x KR135 showed significant SCA effects in desirable direction for green fodder yield, plant height, number of leaves per plant, leaf breadth, number of nodes per plant and stem girth.

Overall, the cross combinations 185A $\mathrm{x}$ RSSV466, 185A x RSSV138-1, PMS71A x RSSV138-1 and PMS71A $x$ RSSV404 exhibited highly significant SCA effects in desirable direction coupled with high per se performance for green fodder yield, and other yield contributing characters viz., days to 50 per cent flowering, plant height, number of leaves per plant, leaf length and leaf breadth (Table 6). These combinations were derived from High $x$ High and Low x High combiners indicating the presence of complementary or dominance gene action in the direction of additive effects of good performer. So, these cross combinations could be used in forage sorghum improvement programme.

\section{Acknowledgement}

I am thankful to ICAR-Indian Institute of Millets Research, Hyderabad and AICRP on Sorghum co-ordinating centers for sharing the seed material which provided a great scope to study forage attributes in sweet sorghum.

\section{References}

Akbari, V.R., Parmar, H.P., Niranjana, M and Nakarani, D.B. 2013. Heterosis and combining ability for green fodder yield and its contributing traits in forage sorghum. The Indian Society of Forage

Research. 38 (3): 156-163.

Anonymous, 2011. Annual Report. All India Coordinated Sorghum Improvement Project, Hyderabad.

Azam, M., Waraich, E.A., Perviaz, A and Nawaz, F. 2010. Response of a newly developed fodder sorghum (Sorghum bicolor L. Moench) variety (F-9917) to NPK application. Pakistan Journal of life sciences. 8 (2): 117-120.

Bhatt, A., and Bhasketi, D.C. 2011. Combining ability studies for green forage yield and its component characters in sorghum (Sorghum bicolor L. Moench) through line $\mathrm{x}$ tester analysis. Pantnagar Journal of Research. 9 (1): 57-60.

Dhananjoy Datta. 2013. Indian Fodder Management towards 2030: A Case of Vision or Myopia. International Journal of Management and Social Sciences Research. 2: 33-41.

Kamdi, S.R., Manjare, M.R and Sushir, K.V. 2011. Combining ability analysis for forage yield and yield contributing characters in sweet sorghum [Sorghum bicolor (L.) Moench]. Mysore Journal of Agricultural Sciences. 45 (4): 837843.

Livestock census - 2012 All India Report, Ministry of Agriculture, Department of Animal husbandry, Dairying and Fisheries, Krishi bhavan, New Delhi.

Mohammed, M.I., 2009. Line x tester analysis across locations and years in Sudanese $\mathrm{x}$ exotic lines of forage sorghum. Journal of Plant Breeding and Crop Science. 1 (9): 311-319.

Poehlman, J.M., Sleper, D.A. 2006. Breeding Field Crops. 5: 297-299.

Reddy, B.V.S., Ramesh, S., Reddy, P.S., Ramaiah, B., Salimath, P.M and Kachapur, R. 2005. Sweet sorghum-a potential alternative raw material for 
bioethanol and bio-energy. Int. Sorghum and Millets Newsl. 46: 79-86.

Tariq, A.S., Akram, Z., Shabbir, G., Khan, K.S and Iqbal, M.S. 2014. Heterosis and combining ability studies for quantitative traits in fodder sorghum (Sorghum bicolor L.). Journal of Agricultural Sciences. 52 (3): 329-337.

\section{How to cite this article:}

Soujanya, T., T. Shashikala and Umakanth, A.V. 2017. Heterosis and Combining Ability Studies in Sweet Sorghum [Sorghum bicolor (L.) Moench] for Green Fodder Yield and Its Contributing Traits. Int.J.Curr.Microbiol.App.Sci. 6(10): 3434-3442. doi: https://doi.org/10.20546/ijcmas.2017.610.405 